\title{
PIK3CA mutations are common in lobular carcinoma in situ, but are not a biomarker of progression
}

Vandna Shah ${ }^{1 \dagger}$, Salpie Nowinski ${ }^{1 \dagger}$, Dina Levi ${ }^{1 \dagger}$, Irek Shinomiya ${ }^{1}$, Narda Kebaier Ep Chaabouni ${ }^{1}$, Cheryl Gillett ${ }^{1}$, Anita Grigoriadis², Trevor A. Graham³ ${ }^{3}$ Rebecca Roylance ${ }^{4}$, Michael A. Simpson ${ }^{5}$, Sarah E. Pinder ${ }^{1}$ and Elinor J. Sawyer ${ }^{1 *}$

\begin{abstract}
Background: Lobular carcinoma in situ (LCIS) is a non-invasive breast lesion that is typically found incidentally on biopsy and is often associated with invasive lobular carcinoma (ILC). LCIS is considered by some to be a risk factor for future breast cancer rather than a true precursor lesion. The aim of this study was to identify genetic changes that could be used as biomarkers of progression of LCIS to invasive disease using cases of pure LCIS and comparing their genetic profiles to LCIS which presented contemporaneously with associated ILC, on the hypothesis that the latter represents LCIS that has already progressed.

Methods: Somatic copy number aberrations (SCNAs) were assessed by SNP array in three subgroups: pure LCIS, LCIS associated with ILC and the paired ILC. In addition exome sequencing was performed on seven fresh frozen samples of LCIS associated with ILC, to identify recurrent somatic mutations.

Results: The copy number profiles of pure LCIS and LCIS associated with ILC were almost identical. However, four SCNAs were more frequent in ILC than LCIS associated with ILC, including gain/amplification of CCND1. CCND1 protein over-expression assessed by immunohistochemical analysis in a second set of samples from 32 patients with pure LCIS and long-term follow up, was associated with invasive recurrence ( $P=0.02$, Fisher's exact test). Exome sequencing revealed that PIK3CA mutations were as frequent as $C D H 1$ mutations in LCIS, but were not a useful biomarker of LCIS progression as they were as frequent in pure LCIS as in LCIS associated with ILC. We also observed heterogeneity of PIK3CA mutations and evidence of sub-clonal populations in LCIS irrespective of whether they were associated with ILC.

Conclusions: Our data shows that pure LCIS and LCIS co-existing with ILC have very similar SCNA profiles, supporting the hypothesis that LCIS is a true precursor lesion. We have provided evidence that over-expression of CCND1 may identify a subgroup of patients with pure LCIS who are more likely to develop invasive disease, in contrast to PIK3CA mutations, which occur too early in lobular tumorigenesis to be informative.
\end{abstract}

Keywords: Lobular carcinoma in situ, Somatic copy number aberrations, CCND1, PIK3CA, Heterogeneity

\footnotetext{
* Correspondence: elinor.sawyer@kcl.ac.uk

${ }^{\dagger}$ Equal contributors

'Division of Cancer Studies, Guy's Hospital, King's College London, London

SE1 9RT, UK

Full list of author information is available at the end of the article
} 


\section{Background}

Lobular carcinoma in situ (LCIS) is a non-invasive breast lesion that is typically found incidentally on biopsy but is also often seen in the presence of invasive lobular carcinoma (ILC), which accounts for 10-15\% of all invasive breast carcinomas. Hwang et al. showed that ILC and coexisting LCIS share many of the same genetic aberrations [1]. Furthermore, Vos et al. demonstrated the presence of the same truncating mutations in $\mathrm{CDH} 1$ and loss of heterozygosity $(\mathrm{LOH})$ of the wild-type E-cadherin in the LCIS component and adjacent ILC [2].

These studies suggest that LCIS is a non-obligate precursor of ILC in a manner analogous to ductal carcinoma in situ (DCIS) preceding invasive ductal carcinoma of no special type (IDC). However, the risk of invasive cancer after LCIS is lower than that with DCIS (2-11 times greater than the risk in the general population, in contrast to DCIS with 20 times greater risk) [3, 4], and the overall rate of progression of pure LCIS to ipsilateral ILC has been shown to be $<10 \%$ ten years after the diagnosis of LCIS $[5,6]$. LCIS is also considered a risk factor for future breast cancer, as not all invasive disease post LCIS presents as ILC and, unlike DCIS, LCIS is also a risk factor for developing invasive cancer in the contralateral breast [7]. Fisher et al. reported that $80 \%$ of invasive disease post LCIS is ILC; however, it is likely that the patients in that study did not represent typical cases of classical LCIS (cLCIS) as many were initially diagnosed as having DCIS, but on pathological review were determined to have LCIS [8]. A more recent study has reported much lower rates of ILC post LCIS (27\%), although this was still higher than the expected $10-15 \%$ [6].

The timescale for the development of invasive carcinoma after an initial diagnosis of LCIS in either breast varies greatly between individuals; one study demonstrated that two thirds of patients developed invasive disease within 15 years; however, another study found that $50 \%$ of patients developed ILC up to 15 to 30 years later. This has led some to argue against LCIS as a non-obligate precursor lesion and to suggest that "pure" LCIS may have a different molecular profile compared to LCIS that coexists with invasive disease, and that the molecular studies cited above have focused on LCIS with associated ILC, rather than pure LCIS.

There are limited studies of pure LCIS but generally these do show similar genetic changes to LCIS associated with ILC, with $16 \mathrm{q}$ loss and $1 \mathrm{q}$ gain being the most common chromosomal abnormalities. Whilst Mastracci et al. suggested that $\mathrm{LOH}$ at $16 \mathrm{q}$ was infrequent in 13 cases of pure LCIS [9], comparative genomic hybridization studies on 17 cases of pure LCIS revealed 16q loss in $88 \%$ of cases, being the sole detected alteration in $29 \%$ [10]. In the latter study, $1 \mathrm{q}$ gain was the second most common change, occurring in $41 \%$ of tumours and in all cases associated with $16 \mathrm{q}$ loss.
There is also evidence of E-cadherin loss in both LCIS and atypical hyperplasia with $\mathrm{CDH} 1$ mutations being common in LCIS, but rare in atypical lobular hyperplasia (ALH) [11].

The increased breast biopsy rate associated with screening mammography has led to an increase in the diagnosis of pure LCIS in postmenopausal women [12] with around $3 \%$ of needle biopsies identifying pure LCIS [13]. Current guidelines for patients with a diagnosis of LCIS highlight the need for increased surveillance of both the affected and contralateral breasts; however, the optimum management of women with pure LCIS is unclear, as not all women with LCIS will develop invasive disease. Currently in the UK patients with pure LCIS do not receive any further treatment and, even if incompletely excised, no further surgery is performed. There is now convincing evidence from large randomized chemoprevention trials, that 5 years of endocrine therapy reduces the risk of invasive disease after a diagnosis of LCIS by $50 \%$; the NASBP-P1 study demonstrated that 5 years of tamoxifen reduced the development of invasive disease after LCIS from $11 \%$ in the control group to $4 \%$ in the tamoxifen-treated group [14]. Similarly 5 years of exemestane reduced invasive disease from 13 to $6 \%$, respectively [15]. However, despite this evidence, the use of chemoprevention for LCIS has not become common practice; seemingly clinicians and patients feel that as many cases of LCIS do not progress to invasive disease, the benefits of chemoprevention do not outweigh the potential side effects. It would therefore be invaluable to have biomarkers to predict the likelihood of progression of LCIS, so that appropriate screening and treatment can be offered.

Current biomarker data in LCIS are very limited [16]. There is some evidence to suggest the risk of subsequent invasive disease is associated with high Ki67 expression [17] and that increased expression of hsa-miR-375 contributes to lobular neoplastic progression [18]. Other studies have shown that the five biomarkers known to be important in invasive breast cancer (oestrogen receptor (ER), progesterone receptor (PgR), c-erbB-2, p53 and Ki-67 expression) do not predict progression of LCIS [19]. One of the problems with studies that have tried to identify biomarkers that predict LCIS recurrence is the small number of cases analysed due to the rarity of pure LCIS.

The aim of the present study was to identify genetic changes that could be used as biomarkers of progression of LCIS to invasive disease. Ideally this should be done in a cohort of patients with pure LCIS, who have progressed to invasive disease, compared to a cohort that have not, but such patients are very rare. We therefore chose to do our discovery phase using patients with pure LCIS and comparing their genetic profiles to patients with LCIS who presented contemporaneously with associated ILC, based on the hypothesis that the latter represents LCIS that has already progressed. 


\section{Methods}

\section{Samples}

Archival cases were identified from the study to investigate the genetics of lobular carcinoma in situ in Europe (GLACIER) (06/Q1702/64). In this study blood samples and formalin-fixed paraffin-embedded (FFPE) tumour samples were available for women with LCIS from 97 hospitals throughout the UK, together with data on known hormonal risk factors for breast cancer. For the discovery phase 30 patients with classic pure LCIS (pure-cLCIS) and 30 with classic LCIS associated with ILC (inv-cLCIS) were selected for SNP-array analysis to assess copy number changes, together with 7 patients with classic LCIS with ILC who had fresh-frozen lesions stored in the KHP Cancer Biobank (NHS REC ref. 12-EE-0493), which were used for whole exome sequencing in order to assess the frequency of mutations in inv-cLCIS.

Following independent review by a breast histopathologist (SEP) to confirm the diagnosis, samples were visually assessed to determine the presence of sufficient malignant tissue for needle dissection under a light microscope (macrodissection). E-cadherin expression was also assessed by immunohistochemical analysis and in all cases Ecadherin staining was absent within the LCIS/ILC with the exception of one pure LCIS sample, which had occasional foci of patchy weak membrane staining.

Up to $2010-\mu \mathrm{m}$ sections were stained using Nuclear Fast Red (Sigma) and macrodissected to separate the LCIS and invasive components. In order to obtain enough DNA to perform these experiments, all the pure-cLCIS foci in a single FFPE block were macrodissected (maximum area $2 \mathrm{~cm}^{2}$ ). In samples from 23 women with inv-cLCIS, this was on the same block as the invasive tumour and in samples from 5 women it was on an adjacent block. Tumour DNA was extracted using the QIAamp DNA FFPE Tissue Kit (Qiagen) for archival specimens and the DNeasy Blood \& Tissue Kit (Qiagen) for fresh-frozen samples. Patient-matched germline DNA was extracted from peripheral blood samples using the Nucleon product chemistry (Tepnel, Manchester, UK). DNA was quantified using Quant-i ${ }^{\mathrm{TM}}$ PicoGreen $^{\bullet}$ dsDNA Assay Kit (Life Technologies).

For the validation of potential biomarkers, we studied samples from 37 patients with pure LCIS, who were diagnosed between 1980 and 2011 and had been followed up for longer than 6 months (median follow up was 81 months, range 34-333). A table summarizing all the clinico-pathological data on the samples used in this study can be found in Additional file 1: Table S1 (discovery set) and Additional file 1: Table S2 (validation set).

\section{SNP arrays}

Samples were hybridised onto the Oncoscan ${ }^{\mathrm{TM}}$ Affymetrix array. The array uses molecular inversion probe (MIP) technology to detect 335,000 markers for genome-wide, allele-specific copy numbers with enhanced coverage of known cancer genes, and which also provides a mutation score for the likelihood of the sample containing a somatic mutation in a series of key cancer genes (http://www.affymetrix.com). A minimum of 200 ng DNA for each sample was used. Labelling and hybridization were outsourced to Affymetrix (Santa Clara, CA, USA).

Raw array data was preprocessed with Nexus 7.5 software (BioDiscovery), applying the SNPRank segmentation algorithm with a minimum of 10 probes per segment. Tumour Aberration Prediction Suite (TAPS) 2.0 [20] was used to determine absolute copy number and for categorisation into: gains (copy number $(\mathrm{CN})=/>3$ ); amplification $(\mathrm{CN}>/=5)$, losses $(\mathrm{CN}=/<1)$; and copy neutral LOH (cnLoH). Samples found to have whole genome duplication were corrected to a diploid state. Frequency plots were made using the copy number package in R 3.0.0 (https://www.bioconductor.org/ packages/release/bioc/html/copynumber.html).

Frequency tables of the resulting somatic copy number aberrations (SCNAs) were generated for pure LCIS, the LCIS associated with ILC, and the paired ILC, and the prevalence of SCNAs were compared using Fisher's exact test. Genomic regions with statistically significant differences $(P<0.05)$ but reported in regions of known HAPMAP copy number variations (CNVs) (http://hapmap.ncbi.nlm. nih.gov/) were removed.

Two-way hierarchical clustering of the pure LCIS, invLCIS, and ILC samples was performed using Manhattan distance and Ward's clustering method in the NMF package in $\mathrm{R}$ (http://nmf.r-forge.r-project.org/aheatmap.html) based on type SCNA (=/>100 probes) occurring on each $\mathrm{p}$ and $\mathrm{q}$ arm. The raw data are available (https://www.ncbi.nlm.nih. gov/geo/query/acc.cgi?acc=GSE88909).

\section{Immunohistochemical analysis (IHC) and fluorescence in situ hybridization (FISH)}

CCND1 FISH was performed using 4- $\mu \mathrm{m}$ tissue sections treated with SPOT-Light ${ }^{\circ}$ Tissue Pretreatment kit (Life Technologies) prior to hybridisation with Abbott Molecular Vysis LSI CCND1 and chromosome 11 enumeration probe kit (Abbott Molecular Inc.) according to the manufacturer's instructions. The 11q13 probe covers a genomic region of $378 \mathrm{~kb}$ and includes the following genes: CCND1, FGF19 and FGF4. CCND1 and chromosome 11 enumeration probe (CEP11) foci were counted in a minimum of 20 cells and an average was determined. The ratio of CCND1 to CEP11 foci was calculated and gain was defined as a ratio of 1.5-2.5 and amplification $>2.5$.

CCND1 IHC was performed on $3-\mu \mathrm{M}$ FFPE tissue sections using clone SP4-R (Ventana Medical Systems Inc.) on an automated staining system (VENTANA BenchMark ULTRA, Roche). CCND1 was scored as described previously by Reis-Filho et al. [21] (equivalent to the Allred scoring system for ER) and based on the proportion of stained 
nuclei (scored $0-5$ : $0=$ none; $1=<1 / 100 ; 2=1 / 100-1 / 10 ; 3$ $=1 / 10-1 / 3 ; 4=1 / 3-2 / 3$ and $5=>2 / 3)$ and intensity $(0-3$ : $0=$ no staining; $1=$ weak staining visible only at high magnification; 2 = moderate staining and $3=$ strong staining visible at low magnification). The two scores were combined to give a total score $(0-8: 0-2=$ low; $3-5=$ intermediate and 6-8 = high).

\section{Whole exome sequencing (WES)}

Libraries were prepared from tumour and paired germline DNA using the SureSelect Human All Exon $50 \mathrm{Mb}$ kit (Agilent) and sequenced on Illumina HiSeq 2000 to a mean depth $>\times 100$. Subsequent analysis was performed using our in-house pipeline; in brief, sequencing reads were aligned to the reference human genome hg19 using NovoAlign (http:// www.novocraft.com/products/novoalign/), Samtools [22] was used to create a pileup file and VarScan2 [23] was used to call somatic mutation and indels, annotated using ANNOVAR [24] and cross-referenced with dbSNP and 1000 Genomes. Somatic mutations were called if there was a minimum of $\times 30$ coverage and the mutation was present in at least $10 \%$ of reads.

\section{Sanger sequencing}

Using standard protocols on the Applied Biosystems 3730xl DNA analyzer with the Finch TV software (Geospiza), Sanger sequencing confirmed the presence of mutations detected by the Oncoscan array or exome sequencing. A restriction enzyme enrichment protocol on stock DNA was used [25] for mutations in exon 9 of PIK3CA (c1624G_A and c1633G_A).

\section{Assessing tumour heterogeneity using the SNP-array}

For regions with a copy number score between 1 and 2 on TAPs analysis, i.e. not being designated as loss by the algorthim but showing some loss visually, we used highly polymorphic microsatellite markers to assess whether these segments represented areas of sub-clonal loss or were just experimental variation in calling of copy number 2, by extracting DNA from different areas of LCIS microdissected using the PALM MicroBeam laser capture microscope (Carl Zeiss). Microsatellite markers on 16q (D16S752) or 6q (D6S1627) were used as a "control", as these regions were designated as loss (copy number 1 ) by TAPs.

Having confirmed that the segments that did not reach the threshold for loss did represent subclonal events, we plotted the transformed $\log 2$ ratio and transformed b-allele frequency (allelic imbalance score from TAPS 2.0 output) for each segment of loss to count the number of these segments that were subclonal per sample. Samples in which the three clusters (absolute copy numbers 1, 2 and 3) could not be defined due to poor quality of the DNA or poor hybridisation were excluded (three cILC, three inv-cLCIS and 5 pure cLCIS samples). The segment with an absolute copy number of 1 with the lowest $\log 2$ ratio and highest allelic imbalance score in a sample was assumed to be a clonal event and to be present in $100 \%$ of the tumour cells (generally $16 \mathrm{q}$ ), and it was used as the reference for copy number 1. The Euclidian distance was calculated from the centroid of all the diploid segments to this reference segment. Next the distance of the remaining segments from the diploid centroid was calculated as a proportion of this reference distance (Additional file 2: Figure S1). A sample-specific threshold was established by examining the distribution of the proportions of the segments considered to be diploid by TAPS output. The threshold for sub-clonality was defined as being outside the $99^{\text {th }}$ percentile of this distribution. Applying this threshold to all the segments considered to be copy number 1 in the TAPS output identified sub-clonal segments.

\section{Results}

SNP array-based copy number analysis of classical LCIS In the discovery set, adequate DNA for hybridization onto the Oncoscan V2 array was extracted from 27/30 samples of pure classic LCIS, and from the 30 samples of classic LCIS associated with ILC, 28 had adequate DNA extracted from the LCIS component and 25 from the ILC component. Frequency plots of gains and losses within each group revealed similar patterns, Fig. 1a.

Pure-cLCIS and inv-cLCIS had remarkably similar SCNAs. There was no evidence that pure-cLCIS was any less genetically re-arranged than inv-cLCIS, with pure LCIS samples not clustering together but scattered throughout the inv-LCIS and ILC samples on hierarchical clustering (Fig. 1b). In fact aneuploidy was more common in the pure-cLCIS group with three cases being triploid, and one case having complex genetic re-arrangements suggestive of chromothripsis; none of the inv-cLCIS cases had evidence of such changes (Additional file 2: Figure S2).

All cLCIS samples, irrespective of whether or not they were associated with ILC, had loss or $\mathrm{cnLOH}$ of $16 \mathrm{q}$ as did $24 / 25$ of the paired cILC. One ILC sample had visual evidence of low-level 16q loss but did not reach the threshold for copy number 1 using the TAPS algorithm, most likely due to normal tissue contamination, as there was no evidence of E-cadherin expression on IHC. As expected, gain or $\mathrm{cnLOH}$ of $1 \mathrm{q}$ was the second most frequent change occurring in 21/27 pure c-LCIS samples (78\%), 25/28 invcLCIS samples (89\%) and 21/25 cILC samples (84\%). These two genetic changes clustered strongly together (Fig. 1b), and were the sole SCNAs in five pure - cLCIS and 3 invcLCIS samples. Other frequent SCNAs that occurred in all subtypes were gain of $16 \mathrm{p}$ (8/27 pure-cLCIS, 5/28 invcLCIS and 9/25 cILC samples) and gain of 19p (9/27 purecLCIS, 6/28 inv-cLCIS and 2/25 cILC samples) and loss of 17p (7/27 pure-cLCIS, 8/28 inv-cLCIS and 12/25 cILC samples). Smaller regions $(<10 \mathrm{Mb})$ of gain were found on 
a
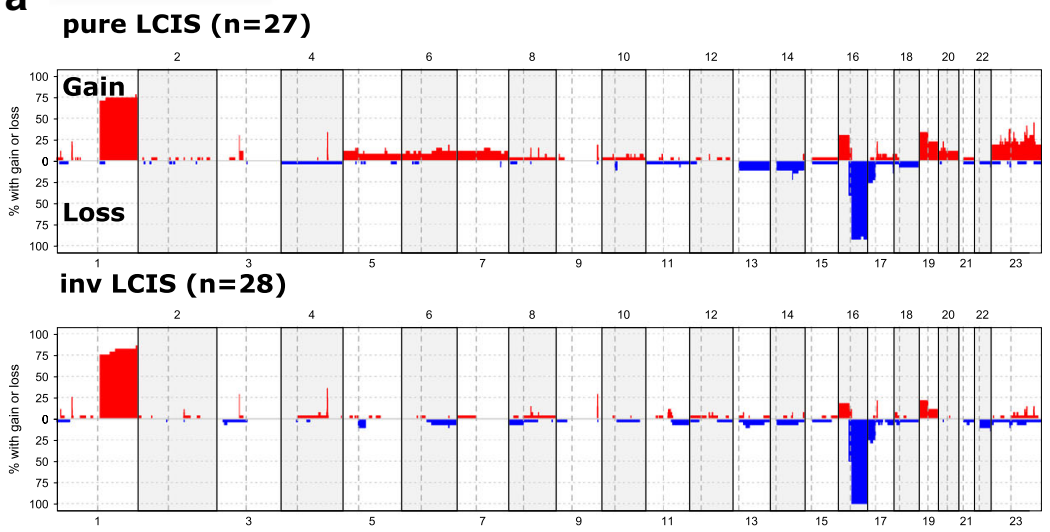

$\operatorname{cILC}(\mathbf{n = 2 5})$

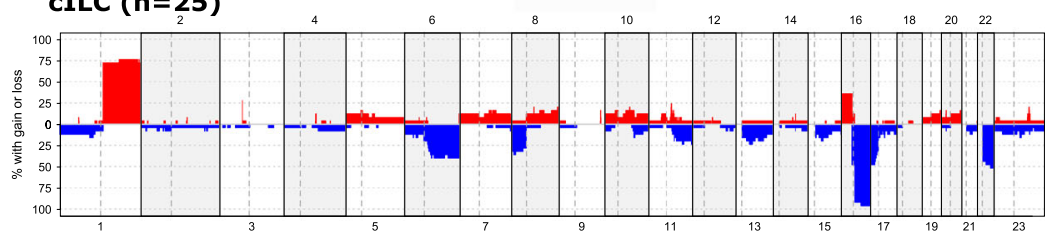

b

Copy Number Lobular Type Clusters

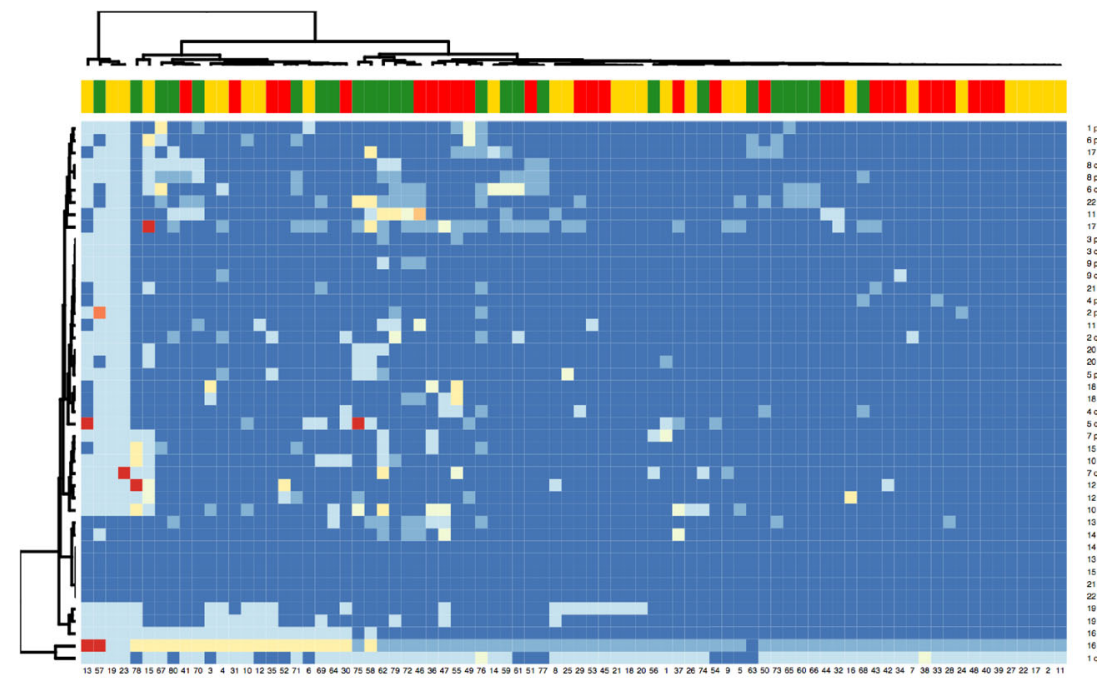

Lobular_Type

$\begin{array}{ll}7 & \text { loss \& gain \& } \mathrm{cnLOH} \\ 6 & \text { gain \& cnLOH } \\ 5 & \text { loss \& cnLOH } \\ 4 & \text { gain \& loss } \\ 3 & \text { cnLOH } \\ 2 & \text { gain } \\ 1 & \text { loss } \\ 0 & \text { diploid normal }\end{array}$

pure cLCIS

CILC

Fig. 1 a Summary of copy number changes in pure classic lobular carcinoma in situ (pure-CLCIS), classic lobular carcinoma in situ associated with invasive lobular cancer (inv-CLCIS) and classic lobular invasive cancer (CILC). b Two-way hierarchical clustering of the pure-CLCIS, inv-CLCIS and CILC based on type of somatic copy number aberration (SCNA) (=/>100 probes) occurring on each $\mathrm{p}$ and $\mathrm{q}$ arm. ( $\mathrm{C} L \mathrm{OH}=$ copy neutral loss of heterozygosity) 
1p34.1, 3p14.1, 9q33.3 and 17q11.2, and of loss were found on 17p13.1 and these occurred with similar frequency in both pure-cLCIS and inv-cLCIS (Additional file 1: Table S3). Of these, only 3p14.1 gain, containing the transcription factor MITF, and loss on 17p13.1 loss (TP53), were found with similar or increased frequency in the paired ILC.

Amplifications (defined as copy number $=/>5$ ) were found in 15 pure-cLCIS samples and 14 inv-cLCIS samples (Additional file 1: Table S4). The most common amplifications in cLCIS were on 1q, encompassing AKT3 (4/27 pure-cLCIS, 4/28 inv-cLCIS and 4/25 ILC samples) and 11q13, encompassing CCND1 (0/27 pure-cLCIS, 2/28 invcLCIS and 5/25 cILC samples).

The only SCNAs that were significantly different between pure-cLCIS and inv-cLCIS were five small regions of gain, more common in pure LCIS, three of which contained a single gene: Xp11 (KLF8), 20p12.1 (MACROD2) and 17q11.2 (RAB11FIP4) (Table 1).

Only one inv-cLCIS/cILC pair clustered together (Fig. 1b); however, this was because in the majority of inv-cLCIS samples the invasive component had the same SCNAs as its paired LCIS but had also acquired additional genetic changes, with SCNAs being more common in cILC than in inv-cLCIS $(P=0.003)$ (Additional file 2: Figure S3). Four regions had an increase in frequency from pure-cLCIS through to inv-cLCIS and cILC: loss of 6q, 8p23 and 22q13 and gain/amplification of 11q13 (Table 1). Among the three regions of loss the most significant increase in frequency came in the transition from inv-cLCIS to cILC.

Gain/amplification of 11q13, was considered potentially the most useful biomarker of LCIS progression, as none of the pure LCIS samples had gain/amplification, but four (two gain, two amplification) of the inv-cLCIS did, with amplification becoming more common in the invasive component. Analysis of the amplicons in our samples revealed that the minimal region of gain/amplification was Chr11: 68961001-70183017, Fig. 2a. This region includes a number of potential oncogenes including CCND1, FGF19, FGF4 and FGF3. FISH using a probe encompassing CCND1, FGF19 and FGF4 confirmed the presence of gain/amplification of 11q13 in all samples with gain/amplification on the SNP array, Fig. 2b. IHC was performed on all samples in the discovery set and this showed that all samples with gain/amplification of 11q13 also had high expression (score 26 ) of cyclin D1. IHC also detected other samples with high protein expression but no evidence of amplification. There was a trend towards higher

Table 1 Summary of somatic copy number aberrations showing a difference in frequency between pure-CLCIS, inv-CLCIS and CILC

\begin{tabular}{|c|c|c|c|c|c|c|c|c|c|}
\hline \multirow[t]{2}{*}{ Chr } & \multirow[t]{2}{*}{ Region } & \multirow[t]{2}{*}{ Genes } & \multirow{2}{*}{$\begin{array}{l}\text { Type of } \\
\text { SCNA }\end{array}$} & \multirow{2}{*}{$\begin{array}{l}\text { PURE } \\
\text { C-LCIS (27) }\end{array}$} & \multirow{2}{*}{$\begin{array}{l}\text { INV- } \\
\text { CLCIS (28) }\end{array}$} & \multirow{2}{*}{$\begin{array}{l}\mathrm{ClLC} \\
(25)\end{array}$} & \multicolumn{3}{|l|}{ P_values } \\
\hline & & & & & & & $\begin{array}{l}\text { Pure } \mathrm{CLCIS} \text { vs } \\
\text { INV-CLCIS }\end{array}$ & $\begin{array}{l}\text { INV-CLCIS VS } \\
\text { CILC }\end{array}$ & $\begin{array}{l}\text { Across all } 3 \\
\text { groups }\end{array}$ \\
\hline 5 & $\begin{array}{l}39436375-39620648 \\
(p 13.1)\end{array}$ & LOC101926940 & Gain & 4 & 0 & 3 & 0.05 & 0.1 & 0.1 \\
\hline 5 & $\begin{array}{l}129741359-131422972 \\
(q 23.3-q 31.1)\end{array}$ & $\begin{array}{l}\text { HINT1, LYRM7, CDC42SE2, } \\
\text { RAPGEF6, FNIP1,MEIKIN, } \\
\text { ACSL6, IL3, CSF2 }\end{array}$ & Gain & 4 & 0 & 2 & 0.05 & 0.2 & 0.09 \\
\hline 6 & $\begin{array}{l}82391438-171115067 \\
(q 14.1-q 27)\end{array}$ & $\begin{array}{l}\text { Many including: MAP3K7, FOXO3, } \\
\text { ESR1, IGF2R, MAP3K4, etc. }\end{array}$ & Loss & 0 & 2 & 10 & 0.5 & 0.007 & 0.00007 \\
\hline 8 & $\begin{array}{l}5412833-8927086 \\
(p 23.2-p 23.1)\end{array}$ & $\begin{array}{l}\text { MCPH1, ANGPT2, AGPAT5, XKR5, } \\
\text { Defensins, FAM66B,SPAG1 } 1 \text { B \&A, } \\
\text { CLDN23, ERI1 }\end{array}$ & Loss & 1 & 2 & 9 & 1 & 0.04 & 0.002 \\
\hline 10 & $\begin{array}{l}75541103-76515425 \\
(q 22.2)\end{array}$ & $\begin{array}{l}\text { CHCHD1,VCL,PLAU,ADK,AP3M1, } \\
\text { CAMK2G, NDST2 }\end{array}$ & Gain & 2 & 0 & 5 & 0.2 & 0.05 & 0.03 \\
\hline 11 & $\begin{array}{l}68961001-71551048 \\
(q 13.3-q 13.4)\end{array}$ & CCND1, MYEOV, FGF4, FGF3 & $\begin{array}{l}\text { Gain } \\
\text { (Amp) }\end{array}$ & 0 & $4(2)$ & $6(5)$ & 0.1 & 0.1 & 0.02 \\
\hline 17 & $\begin{array}{l}29779560-29899917 \\
(q 11.2)\end{array}$ & RAB11FIP4 & $\begin{array}{l}\text { Gain } \\
\text { (Amp) }\end{array}$ & $5(1)$ & 0 & 0 & 0.02 & 0.005 & 0.005 \\
\hline 18 & $\begin{array}{l}\text { 727180-742194 } \\
(\mathrm{p} 11.32)\end{array}$ & YES1 & $\begin{array}{l}\text { Gain } \\
\text { (Amp) }\end{array}$ & $7(1)$ & 2 & 0 & 0.07 & 0.02 & 0.02 \\
\hline$x$ & $\begin{array}{l}\text { 55670623-57693679 } \\
\text { (p11.21) }\end{array}$ & KLF8 & Gain & 10 & 0 & 1 & 0.0003 & 0.00004 & 0.00004 \\
\hline 20 & $\begin{array}{l}\text { 14695735-15225214 } \\
\text { (p12.1) }\end{array}$ & MACROD2 & Gain & 6 & 0 & 1 & 0.01 & 0.006 & 0.006 \\
\hline 22 & $\begin{array}{l}47751337-51304566 \\
(q 13.31-q 13.3)\end{array}$ & $\begin{array}{l}\text { BRD1, HDAC10, MAPK12. } \\
\text { MAPK11 ... }\end{array}$ & Loss & 2 & 4 & 13 & 0.6 & 0.005 & 0.0003 \\
\hline
\end{tabular}

Pure-CLCIS pure classic lobular carcinoma in situ, inv-CLCIS classic lobular carcinoma in situ associated with invasive lobular cancer, cILC classic invasive lobular cancer, Chr chromosome, SCNA somatic copy number aberration 


\section{a}

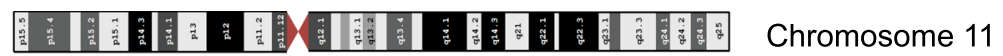

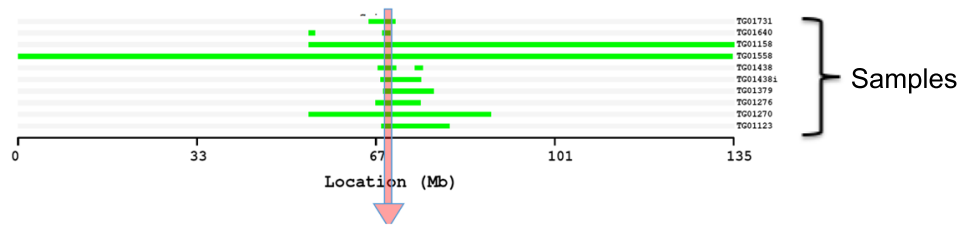

Genes in minimal region of overlap

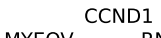

MYEOV RNU6-1175P

IFITM9P ANO1

ORAOV1 ANO1-AS1

FGF19 FADD

FGF4

ANO1-AS2 MIR548K

b

FISH Chr11 SNP Array IHC

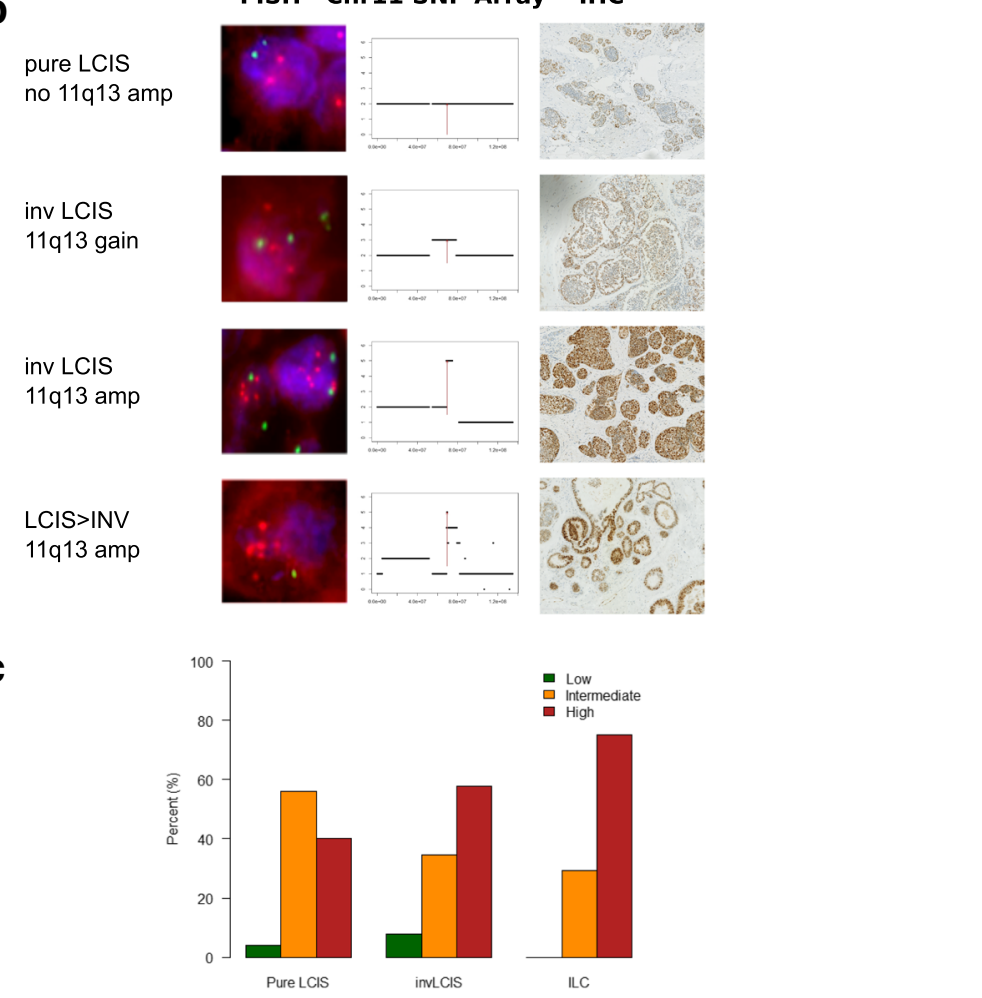

Fig. 2 a Minimal region of gain/amplification on 11q; b correlation of copy number change at 11q13 with fluorescence in situ hybridization (FISH) using the probe for CCND1 and Cyclin D1 immunohistochemical analysis (IHC); c frequency of Cyclin D1 expression as measured by IHC in the discovery set. (SNP = single nucleotide polymorphism, LCIS = lobular carcinoma in situ, Inv LCIS = lobular carcinoma in situ associated with invasive lobular cancer)

cyclin D1 expression from pure LCIS through to invCLCIS and ILC $(P=0.07)$ (Fig. 2c).

In order to assess whether expression of cyclin D1 could be a potential marker of progression, IHC was performed on a different set of samples from 32 patients from the GLACIER study, who had long term follow up and FFPE blocks available, and had not received chemoprevention or had undergone mastectomy (Additional file 1: Table S2). Of these 32 patients, 8 had developed invasive disease with a median time to recurrence of 69 months (range 34-175).
Four had developed ipsilateral recurrence (two ILC, one IDC, one ILC and IDC) and four, contralateral invasive disease (one tubular carcinoma, one IDC and two ILC) (Additional file 1: Table S2a). The latter were excluded from the analysis as they do not represent direct clonal progression of LCIS. Cyclin D1 protein overexpression was associated with recurrence ( $P=0.02$, Fisher's exact test) with high expression in $4 / 4$ samples from patients with pure LCIS that progressed to ipsilateral invasive disease, compared to only $8 / 24$ samples from patients with pure LCIS, 
with no evidence of recurrence after a minimum of 60 month follow up.

IHC had a positive predictive value (PPV) of $33 \%$ and negative predictive value (NPV) of $100 \%$. FISH was only performed on samples in the validation set with high cyclin D1 expression on IHC, as our data and others [26] have shown correlation between amplification and high expression on IHC. One of the four patients that developed ipsilateral invasive disease (Fig. 2c-iv) had amplification of CCND1 and subsequently developed ILC, which was treated with mastectomy and endocrine therapy but recurred 2 years later in the mastectomy scar. The invasive recurrence also had CCND1 amplification. One of the 24 patients with long-term follow up and no evidence of recurrence had evidence of CCND1 gain ( $P=0.27$, Fisher's exact test).

\section{Mutation analysis}

Seven fresh-frozen samples of classic ILC with associated LCIS were selected for whole exome sequencing (WES) and the two components were macrodissected under the light microscope. Adequate DNA for WES of both the ILC and LCIS components was obtained from only one patient. In the remaining samples three had adequate DNA from the LCIS component and three from the ILC.

In the single paired LCIS-ILC sample, 13 mutations were shared between the two components, including two PIK3CA mutations and one truncating $C D H 1$ mutation, a frameshift mutation in COX15 and stop-gain in DOCK2 (Additional file 1: Table S5a). There was no evidence that these mutated genes targeted a particular biological pathway (http://geneontology.org/). The ILC component had 23 mutations not found in the LCIS component, of which 5 were transcription factors (RB1, ARID4A, VGLL3, ZNF341 and SIX1) (Additional file 1: Table S5b). Similarly the LCIS component had mutations in 10 genes, not found in the ILC component, suggesting there are also driver and passenger mutations at the pre-invasive stage (Additional file 1: Table S5c).

Analysis of the pooled exome sequencing data from all samples revealed that $C D H 1$ and PIK3CA were the most common somatic mutations, which co-existed in $3 / 4$ LCIS and 3/4 ILC samples (Table 2). The finding that PIK3CA mutations were as common as $C D H 1$ mutations in LCIS was surprising and we therefore sequenced the commonest mutations in exon 9 and 20 of PIK3CA (c3140A > G, c3140A > T, c1624G > A, c1633G > A, c125 $8 \mathrm{~T}>\mathrm{C}, \mathrm{c} 1636 \mathrm{C}>\mathrm{A}$ ) in the same samples that underwent Oncoscan array analysis (27 pure-cLCIS and 28 inv-cLCIS samples) to assess their frequency and determine whether PIK3CA mutations could be used as a biomarker for LCIS progression. Due to the limited amount of DNA, the Oncoscan MIP array PIK3CA mutation score was used as a guide as to which mutations to assess in each sample. All PIK3CA mutations with an Oncoscan score $>4.5$ were
Table 2 Whole exome sequencing of four invasive lobular cancer (ILC) samples and four lobular carcinoma in situ (LCIS) samples (one paired): mutations occurring in two or more samples

\begin{tabular}{|c|c|c|c|c|}
\hline Gene & Chrom & $\begin{array}{l}\text { Number of ILC } \\
\text { samples with } \\
\text { mutation }\end{array}$ & $\begin{array}{l}\text { Number of LCIS } \\
\text { samples with } \\
\text { mutation }\end{array}$ & Total \\
\hline HSPG2 & 1 & 1 & 1 & 2 \\
\hline ROR1 & 1 & 1 & 1 & 2 \\
\hline SMG7 & 1 & 1 & 1 & 2 \\
\hline TSSC1 & 2 & 2 & 0 & 2 \\
\hline PIK3CA & 3 & 3 & 3 & 6 \\
\hline DOCK2 & 5 & 1 & 1 & 2 \\
\hline HAND1 & 5 & 2 & 0 & 2 \\
\hline UTP23 & 8 & 2 & 0 & 2 \\
\hline CACNB2 & 10 & 1 & 1 & 2 \\
\hline COX15 & 10 & 1 & 1 & 2 \\
\hline OR56B1 & 11 & 1 & 1 & 2 \\
\hline DDX11 & 12 & 1 & 1 & 2 \\
\hline PROSER1 & 13 & 1 & 1 & 2 \\
\hline TPTE2 & 13 & 1 & 1 & 2 \\
\hline PAK6 & 15 & 2 & 0 & 2 \\
\hline $\mathrm{CDH} 1$ & 16 & 3 & 3 & 6 \\
\hline PTRF & 17 & 1 & 1 & 2 \\
\hline ATP11C & $x$ & 1 & 1 & 2 \\
\hline ATRX & X & 1 & 1 & 2 \\
\hline
\end{tabular}

sequenced using Sanger sequencing. Sanger sequencing DNA extracted from macrodissected samples did not detect any mutations; however, the use of restriction enzymes or microdissection of small areas of tissue using the laser capture microscope (LCM) to minimise normal tissue contamination, confirmed those mutations (Fig. 3).

In classic cases, $5 / 27$ pure-cLCIS samples (18.5\%) and 6/ 28 inv-cLCIS samples (21.5\%) had PIK3CA mutations. In the latter group all of the mutations present in the LCIS were also present in the paired ILC, with the exception of two samples. One contained three different PIK3CA mutations in the LCIS component and only two were transferred to the paired ILC, and the other did not have enough DNA to test the ILC component (Table 3).

These findings suggest that like CDH1, PIK3CA mutations might be early events in lobular tumourigenesis, but are not a potential biomarker for progression from LCIS to ILC.

\section{Assessing tumour heterogeneity using Oncoscan MIP array data}

Exome sequencing revealed that mutations occurred in $10-24 \%$ of the reads in the LCIS samples and in 10-40\% of the ILC samples. As these samples were macrodissected it is highly likely that there was significant normal tissue contamination, particularly in the ILC samples due to the 
a

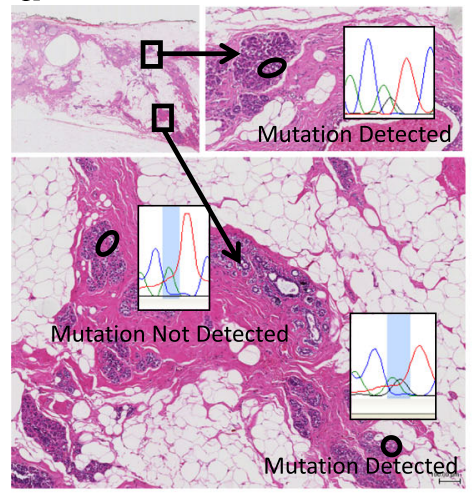

b

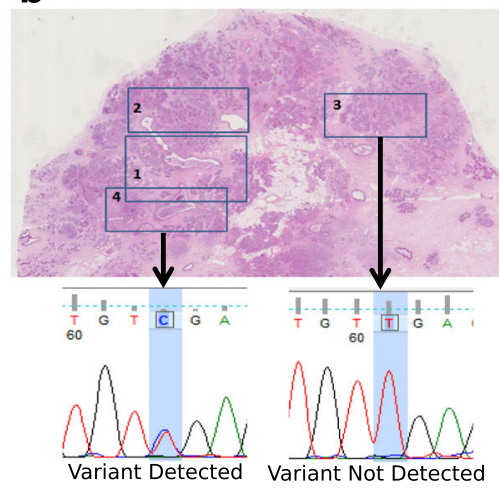

C

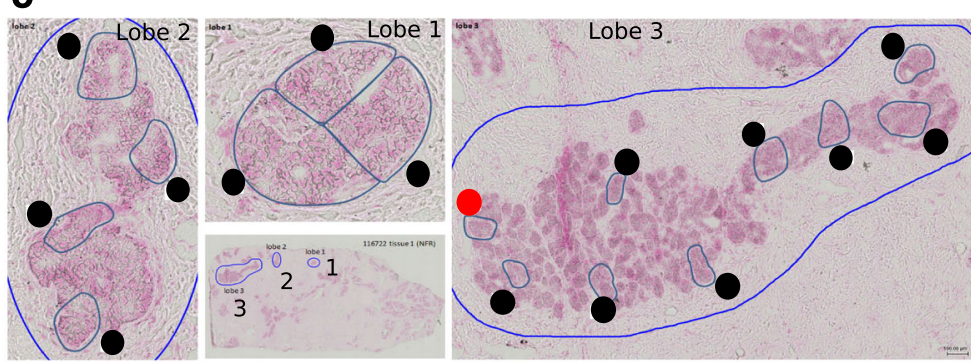

- Heterozygous Variant Detected

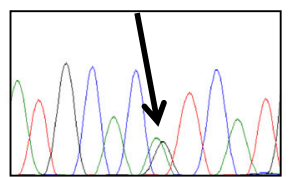

Homozygous

Variant Detected

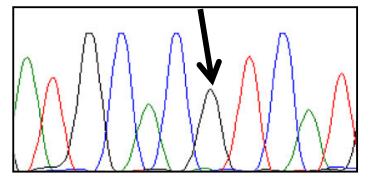

Fig. 3 a Heterogeneity of PIK3CA mutations in two pure classic lobular carcinoma in situ (pure-CLCIS) samples; b heterogeneity of ERBB2 mutation within the LCIS component but not in invasive lobular carcinoma (ILC); c heterozygous and homozygous PIK3CA mutations in invasive CLCIS associated with ILC (inv-CLCIS)

characteristic single cell file infiltration of breast stroma seen in this subtype of carcinomas. However it is unlikely that there was $80 \%$ normal tissue contamination in the LCIS samples and we therefore tested the hypothesis that heterogeneity within the LCIS could also be contributing to the low number of reads with mutations. We sequenced mutations from multiple areas within selected samples microdissected by LCM (two to six areas). In two pure-cLCIS samples there was evidence of heterogeneity of the PIK3CA mutation, Fig. 3a. In a sample with an ERBB2 mutation there was evidence of heterogeneity within the in situ component but not in ILC (Fig. 3b), and in one inv-cLCIS

Table 3 Frequency of PIK3CA mutations in classic lobular carcinoma in situ (LCIS)/invasive lobular cancer (ILC)

\begin{tabular}{llll}
\hline Mutation (total number of cases) & Pure LCIS $(27)$ & inv-LCIS $(28)$ & ILC (25) \\
\hline PIK3CA_pH1047R_c3140A_G & $3\left(1558,1717,04078^{\mathrm{a}}\right)$ & $3(1731,1965,1604)$ & $2^{\mathrm{a}}(1731,1604)$ \\
& & & $1965-\mathrm{no}$ tissue left \\
PIK3CA_pH1047R_c3140A_T & 0 & 0 & 0 \\
PIK3CA_pE542K_c1624G_A & $1(1339)$ & $3(1063,1604,1640)$ & $3(1063,1604,1640)$ \\
PIK3CA_pE545K_C1636C_A & 0 & 0 & 0 \\
PIK3CA_pE545K_c1633G_A & $1(1078)$ & $2(1270,1604)$ & $2(1270,1126)$ \\
Total number of cases with PIK3CA Mutations & 5 & 6 & 6
\end{tabular}

\footnotetext{
${ }^{a}$ sample IDs (italics represent samples with multiple PIK3CA mutations)
} 
sample there was evidence of heterozygous and homozygous PIK3CA mutations (Fig. 3c).

We found further evidence of genetic heterogeneity within LCIS when analysing the SNP-array data. Although the majority of the SCNAs reached the threshold for loss (copy number 1) we also identified segments that did not reach these thresholds (Fig. 4a). In order to ascertain whether these regions could represent sub-clones we used highly polymorphic microsatellite markers for each of the heterogeneous regions (D15S1005 and D15S1038, D11S897, D3S2409) and performed standard LOH analysis on DNA extracted from different areas of microdissected LCIS. Microsatellite markers on 16q (D16S752) or 6q (D6S1627) were used as the control, as these regions reached the threshold for loss.

The microsatellite analysis confirmed that where SCNAs did not reach the threshold for loss in LCIS there was evidence of heterogeneity, with $\mathrm{LOH}$ occurring in $60-80 \%$ of the microdissected regions (Fig. 4b). Following confirmation that these regions did represent sub-clones, we calculated the frequency of such segments in the different subsets of lobular cancer using the SNP-array data as described in "Methods". There was a non-significant trend for the number of samples with sub-clones to increase from pure LCIS to ILC $(P=0.08)$ in the classic form of the disease.

\section{Discussion}

Our study confirms the finding of previous studies that LCIS has the same molecular changes as co-existing ILC. We have also shown that pure LCIS and LCIS associated with ILC (inv-LCIS) have very similar SCNAs, supporting the hypothesis that pure LCIS is a precursor lesion. Only five regions were significantly different between purecLCIS and inv-cLCIS and these were all more common in pure LCIS, so did not represent markers of progression. Three of the regions were small, containing just one gene: chr17 - RAB11FIP4; chr20 - MACROD2, overexpression of this gene has been implicated in oestrogen-independent growth [27]; and chrX - KLF8, oncogenic in ovarian but not breast cancer cell lines [28]. There was also a similar region on chr18, that was more common in both pure and inv-cLCIS compared to ILC, containing YES1, a SRC proto-oncogene that has recently been identified as a possible therapeutic target in basal breast cancer [29]. It is possible that these regions contain genes that hinder development of the invasive phenotype. A similar finding was made in DCIS (albeit with different genomic regions) and the authors hypothesized that these regions could contain genes that provide a selective advantage under local conditions but also inhibit invasion [30].

We identified four SCNAs that increased in frequency from pure cLCIS to inv-cLCIS and finally ILC: loss of 6q14.1-27, 8p23.2-23.1, and 22q13.31-13.33 and gain of 11q13.3. For the three regions of loss the most significant increase in frequency came in the transition from invcLCIS to cILC. Both $6 \mathrm{q}$ and 8p23 loss have also been found to be more common in invasive ductal carcinoma (IDC) than in paired DCIS [30] suggesting that there may be tumour suppressor genes in these chromosomal regions important in the transition of in situ to invasive carcinoma in both DCIS and LCIS. A recent study integrating SCNAs, promoter methylation and gene expression profiles in luminal B breast cancers showed that $88 \%$ of the potential tumour suppressor genes were located on 6q [31]. Deletions of $8 p$ have been described in 50\% of IDC and $37 \%$ of lobular cancers [32] and the minimal region of loss in our study (8p23.2-23.1) includes $M C P H 1$, a potential tumour suppressor gene $[33,34]$ encoding a DNA damage response protein which has also been implicated in breast cancer predisposition [35]. Similarly, loss of 22q13 has been described in IDC (not present in paired DCIS) and ILC (but also present in paired LCIS) [36, 37].

Gain/amplification of 11q13.3 was considered the most useful SCNA to take forward as a potential practical biomarker of LCIS progression as unlike the regions of loss, the main increase in frequency occurred between the purecLCIS and inv-cLCIS sub-groups. There was also evidence of increasing amplitude of copy number gain between the paired inv-cLCIS and ILC samples. The region contains CCND1 and other potential oncogenes such as FGF3, FGF4 and MYEOV and there is good evidence that this region is relevant to breast cancer [38]. Two large studies of DCIS (approximately 400 patients) have reported amplification of CCND1 in $10-12.6 \%$ of patients with pure DCIS and $14.8-17.4 \%$ of patients with DCIS associated with invasive breast cancer, with the majority of patients having amplification in the paired invasive component $[39,40]$. In a much smaller series of 20 patients with florid LCIS (a subtype putatively more likely to associated with invasive disease) $25 \%$ had CCND1 amplification [41]. This, together with the finding that CCND1 amplification is usually homogeneous within breast carcinomas, suggests that it is an early event in the development of some breast cancers [42].

Other studies have identified correlation between overexpression of cyclin D1 and amplification of 11q13 and this over-expression has been associated with an increased risk of recurrence in ILC [43]. One early study of ILC suggested that cyclin D1 protein overexpression may play a role in the transition of LCIS to ILC, as the majority of ILC samples had over-expression but with little evidence in surrounding LCIS, although the number of patients with LCIS was not stated.

Our data support their finding that cyclin D1 overexpression is important in the transition of LCIS to ILC, but we have also shown that some cases of LCIS also overexpress cyclin D1 and this may be a marker of progression 
a

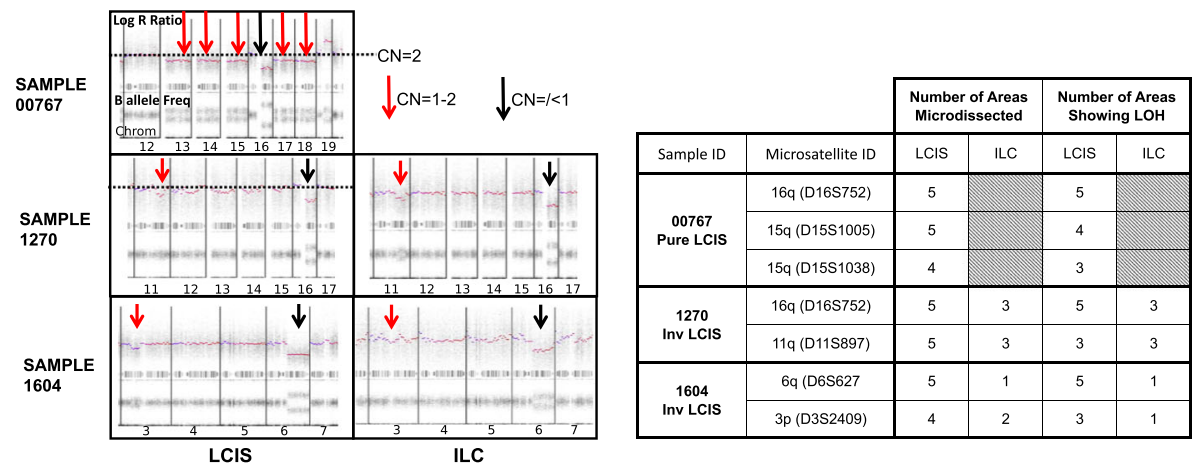

b
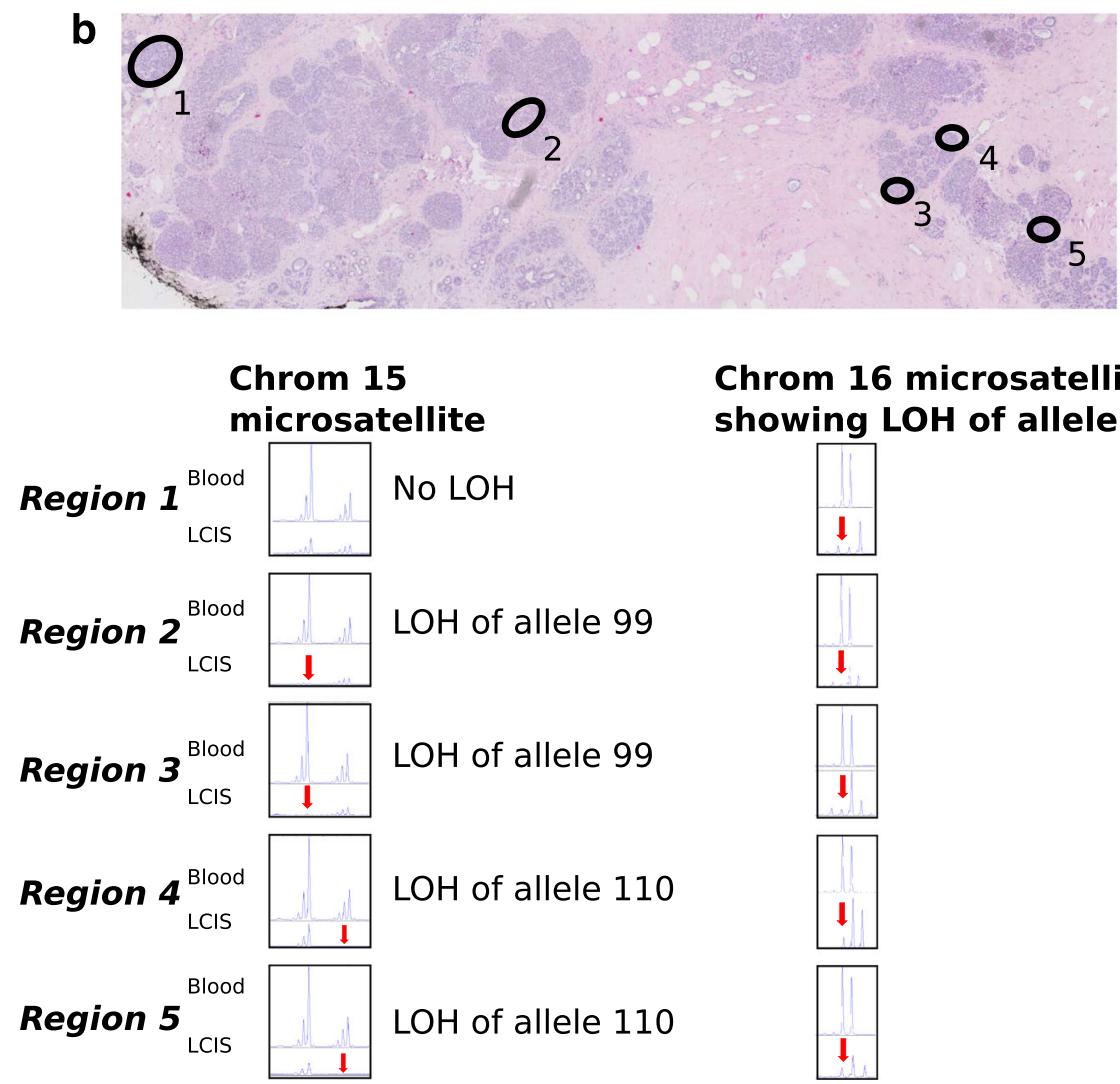

Fig. 4 a Sub-clonal segments on copy number analysis; b microsatellite markers confirm that sub-clonal segments on copy number analysis show heterogeneous loss. ( $\mathrm{LOH}=$ loss of heterozygosity)

to ILC [44]. In a genome-driven classification of over 7500 breast tumours, amplification of 11q13.3 was associated with a sub-group of ER-positive breast tumours with a poor prognosis and chemo-resistance. This sub-group accounted for only $3.1 \%$ of tumours in this series of mainly IDC [45]. However, in our study $24 \%$ of cILC had 11 q13.3 amplification in keeping with other series showing 11q13.3 amplification is more frequent in ILC than IDC [46]. In our validation set, albeit very small, we found that cyclin D1 over-expression may identify a subset of pure LCIS that is likely to progress to ILC. As around $40 \%$ of ILC have high expression of cyclin D1 [43], this represents a significant subset of ILC.

Exome sequencing of this small number of LCIS and ILC did not identify any potential biomarkers of LCIS progression. It did, however, show that activating PIK3CA mutations are as common in LCIS as $C D H 1$ mutations. Activating PIK3CA mutations are well-described in both ILC and IDC, occurring in $48 \%$ of ILC (as the second most common mutations after $C D H 1)$ and $33 \%$ of IDC $[47,48]$. 
PIK3CA mutations have previously been reported by Christgen et al. [49] in 1/3 patients with LCIS associated with ILC and by Sakr et al. [48] in 7/19 patients. We confirmed the frequency of PIK3CA mutations in a larger set of LCIS by Sanger sequencing, which revealed no difference in the frequency of PIK3CA mutations in inv-cLCIS compared to pure-cLCIS. The only other study to assess PIK3CA mutations in pure LCIS was by Sakr et al. who found no evidence of PIK3CA mutations in three patients with pure LCIS after targeted sequencing [48]. The frequency of PIK3CA mutations in ILC in our study is lower than that reported by The Cancer Genome Atlas (TCGA) and Sakr et al.; however, this is likely to be because both those studies used next-generation sequencing to assess the whole gene, whereas we targeted only the common mutations.

So, although they are not a useful biomarker of LCIS progression, PIK3CA mutations are an early event in lobular tumorigenesis leading to abnormal proliferation of the breast epithelium, but importantly, they do not appear to be the critical event leading to invasive malignancy. This is supported by the findings of Ang et al. who identified frequent PIK3CA mutations in non-invasive proliferative breast lesions including DCIS, inv-LCIS and one case of pure LCIS [50, 51].

A comprehensive analysis of 127 ILC by the TCGA has shown that CDH1, PIK3CA, TBX3, FOXA1 and RUNX1 are the most commonly mutated genes [47]. We found no evidence of TBX3, FOXA1 or RUNX1 mutations in the four LCIS samples on which we performed exome sequencing, and identified only one TBX3 mutation in the four ILC samples. The only other known driver gene mutated in LCIS was ATRX, frequently mutated in neuroblastoma, low-grade glioma and glioblastoma but not common in breast cancer. In the present series we report splice site mutation in two additional well-known drivers of cancer, MAP2K4 and RB1 in ILC, but not LCIS,

The TCGA data also showed that AKT signalling is strongly activated in ILC and homozygous losses of the PTEN locus (10q23) occurred in 6\% of ILC [47]. We found no evidence of homozygous deletions of 10q23 in LCIS in our samples. One case of ILC did have a homozygous deletion at the PTEN locus, whilst this was not evident in the paired LCIS, suggesting it is a later event in lobular tumourigenesis. Interestingly the region encompassing $A K T 3$ was the locus most frequently amplified in both pure CLCIS and inv-cLCIS and paired cILC. So, although PIK3CA mutations do not appear to be the trigger for malignant transformation in lobular cancer, it is possible that progression of LCIS may be related to acquisition of mutations or alterations in other components of the PI3K/Akt pathway; for example, expression profiling studies have shown that PIK3R1 is significantly downregulated in the stepwise progression from normal epithelium to LCIS to ILC [52].
With the advent of next-generation sequencing intratumoural heterogeneity has been found to be widespread in invasive cancer; however, there are few data on the intra-tumoural heterogeneity of in situ breast cancers. We developed a relatively crude method to assess heterogeneity using SNP arrays and this clearly showed that subclonal SCNAs increase in frequency from in situ to invasive lobular carcinoma. It remains to be seen whether a more sensitive measure of clonal diversity could be used as a biomarker of progression to invasive disease, as it is in Barrett's oesophagus [53, 54]. Of interest, we have also shown evidence of passenger mutations in LCIS not transmitted to the invasive component, suggesting that, like invasive disease, there is an early sub-clone expansion process [55], with at least one acquiring critical mutations and developing into invasive disease. Driver mutations that are sub-clonal in the pre-invasive state then become clonal in the invasive stage.

\section{Conclusions}

In conclusion our data have shown that pure LCIS and LCIS co-existing with ILC have very similar SCNA profiles, suggesting that pure LCIS is not intrinsically a different process that is less likely to develop into invasive disease. We have identified four SCNAs that are important in the transformation of LCIS to ILC and provided evidence that over-expression of cyclin D1 may identify a subgroup of LCIS more likely to develop invasive disease. This needs confirming in larger studies, although this will be challenging, as there are few series with long-term follow up of pure LCIS. We have also shown that PIK3CA mutations are common in LCIS and that there is genetic heterogeneity within LCIS, just as in ILC.

\section{Additional files}

\begin{abstract}
Additional file 1: Table S1a. Clinico-pathological features of the discovery set - pure LCIS. Table S1b. Clinico-pathological features of the discovery set inv-LCIS and ILC. Table S2a. Validation set: characteristics of the eight pure LCIS tumours that recurred. Table S2b. Validation set: characteristics of pure LCIS tumours that did not recur. Table S3. Common regions of gain /loss $<10$ $\mathrm{Mb}$ in size in classic lobular subtypes. Table S4. Regions of amplification occurring in more than one sample. Table S5a. Somatic mutations identified by whole exome sequencing in both the LCIS and ILC components in a single paired case. Table S5b. Somatic mutations identified by whole exome sequencing in ILC but not the LCIS component in a single paired case. Table S5c. Somatic mutations identified by whole exome sequencing in LCIS but not the ILC component in a single paired case. (DOCX $46 \mathrm{~kb}$ )
\end{abstract}

Additional file 2: Figure S1. Example of subclonal loss calculation using SNP array. Figure S2. Pure C-LCIS showing anueploidy and chromothripsis. Figure S3. Proportion of SCNA breakpoints in different subtypes of lobular cancer. (PDF 2813 kb)

\section{Abbreviations}

CILC: classic invasive lobular cancer; CLCIS: classic lobular carcinoma in situ; cnLOH: copy number loss of heterozygosity; CNV: copy number variation; DCIS: ductal carcinoma in situ; ER: oestrogen receptor; FFPE: formalin-fixed paraffin-embedded tissue; FISH: fluorescence in situ hybridization; IDC: invasive 
ductal carcinoma; ILC: invasive lobular cancer; inv-CLCIS: classic lobular carcinoma in situ associated with ILC; LCIS: lobular carcinoma in situ; LOH: loss of heterozygosity; MIP: molecular inversion probe; pILC: pleomorphic invasive lobular cancer; pLCIS: pleomorphic lobular carcinoma in situ; SCNA: somatic copy number aberration; SNP: single nucleotide polymorphism; TAPS: Tumour Aberration Prediction Suite; TGCA: The Cancer Genome Atlas; WES: whole exome sequencing

\section{Acknowledgements}

This research was supported by the National Institute for Health Research (NIHR) Biomedical Research Centre at Guy's and St Thomas' National Health Service (NHS) Foundation Trust and King's College London. The views expressed are those of the author(s) and not necessarily those of the NHS, the NIHR or the Department of Health. King's Health Partners Cancer Biobank is supported by the Department of Health via the National Institute for Health Research (NIHR) comprehensive Biomedical Research Centre award and the Experimental Cancer Centre at King's College London.

\section{Funding}

Sample and data collection were funded by Cancer Research UK. Analysis was funded by Breast Cancer Now, the Rosetrees Trust, Guys \& St Thomas' Charity (CanHelp) and the National Institute for Health Research (NIHR) Biomedical Research Centre based at Guy's and St. Thomas' NHS Foundation Trust and King's College London.

\section{Availability of data and materials}

The datasets supporting the conclusions of this article are included within the article and its additional files. Raw SNP array data be accessed at: https:// www.ncbi.nlm.nih.gov/geo/query/acc.cgi?acc=GSE88909.

\section{Authors' contributions}

EJS conceived and designed the experiment. EJS, RR and CG performed sample collection. NKEC analysed clinical data. SP performed histopathological review. VS, DL and IS performed experiment. SN performed data analysis. AG and TAG advised on data analysis. MAP performed exome sequencing. EJS prepared the manuscript preparation. All authors performed critical review of the manuscript. All authors read and approved the final manuscript.

\section{Authors' information}

See title page.

\section{Competing interests}

The authors declare that they have no competing interests.

\section{Consent for publication}

Not applicable.

\section{Ethics approval and consent to participate}

GLACIER study: MREC 06/Q1702/64. KHP Tissue Bank: NHS REC ref. 12-EE-0493.

\section{Author details}

'Division of Cancer Studies, Guy's Hospital, King's College London, London SE1 9RT, UK. 'Breast Cancer Now Unit, Research Oncology \& Cancer Epidemiology, Guy's Hospital, King's College London, London SE1 9RT, UK. ${ }^{3}$ Evolution and Cancer laboratory, Centre for Tumour Biology, Barts Cancer Institute, Queen Mary University of London, London, UK. ${ }^{4}$ Department of Oncology, UCLH Foundation Trust, London NW1 2PG, UK. ${ }^{5}$ Medical and Molecular Genetics, Guy's Hospital, King's College London, London, UK.

\section{Received: 11 July 2016 Accepted: 1 December 2016}

\section{Published online: 17 January 2017}

\section{References}

1. Hwang ES, Nyante SJ, Yi Chen Y, Moore D, DeVries S, Korkola JE, Esserman $\sqcup$, Waldman FM. Clonality of lobular carcinoma in situ and synchronous invasive lobular carcinoma. Cancer. 2004;100(12):2562-72.

2. Vos CB, Cleton-Jansen AM, Berx G, de Leeuw WJ, ter Haar NT, van Roy F, Cornelisse CJ, Peterse JL, van de Vijver MJ. E-cadherin inactivation in lobular carcinoma in situ of the breast: an early event in tumorigenesis. Br J Cancer. 1997;76(9):1131-3.

3. Wärnderg F, Yuen J, Holmberg L. Risk of subsequent invasive breast cancer after breast carcinoma in situ. Lancet. 2000;355(9205):724-5.
4. Collins LC, Tamimi RM, Baer HJ, et al. Outcome of patients with ductal carcinoma in situ untreated after diagnostic biopsy: results from the Nurses' Health Study. Cancer. 2005;103(9):1778-84.

5. Christgen M, Steinemann D, Kühnle E, Länger F, Gluz O, Harbeck N, Kreipe H. Lobular breast cancer: clinical, molecular and morphological characteristics. Pathol Res Pract. 2016;212(7):583-97.

6. King TA, Pilewskie M, Muhsen S, Patil S, Mautner SK, Park A, Oskar S, GueriniRocco E, Boafo C, Gooch JC, De Brot M, Reis-Filho JS, Morrogh M, Andrade VP, Sakr RA, Morrow M. Lobular carcinoma in situ: a 29-year longitudinal experience evaluating clinicopathologic features and breast cancer risk. J Clin Oncol. 2015;33(33):3945-52.

7. Chuba PJ, Hamre MR, Yap J, Severson RK, Lucas D, et al. Bilateral risk for subsequent breast cancer after lobular carcinoma-in-situ: analysis of surveillance, epidemiology, and end results data. J Clin Oncol. 2005;23(24):5534-41.

8. Fisher ER, Land SR, Fisher B, Mamounas E, Gilarski L, et al. Pathologic findings from the NSABBP: twelve-year observations concerning lobular carcinoma in situ. Cancer. 2004;100(2):238-44.

9. Mastracci TL, Shadeo A, Colby SM, Tuck AB, O'Malley FP, Bull SB, Lam WL, Andrulis IL. Genomic alterations in lobular neoplasia: a microarray comparative genomic hybridization signature for early neoplastic proliferation in the breast. Genes Chromosomes Cancer. 2006;45(11):1007-17.

10. Etzell JE, Devries S, Chew K, Florendo C, Molinaro A, Ljung BM, Waldman FM. Loss of chromosome $16 \mathrm{q}$ in lobular carcinoma in situ. Hum Pathol. 2001;32:292-6.

11. Mastracci TL, Tjan S, Bane AL, O'Malley FP, Andrulis IL. E-cadherin alterations in atypical lobular hyperplasia and lobular carcinoma in situ of the breast. Mod Pathol. 2005;18(6):741-51.

12. $\mathrm{Li} \mathrm{Cl}$, Daling JR, Malone KE. Age-specific incidence rates of in situ breast carcinomas by histologic type, 1980 to 2001. Cancer Epidemiol Biomarkers Prev. 2005:14(4):1008-11.

13. Susnik B, Day D, Abeln E, Bowman T, Krueger J, Swenson KK, Tsai ML, Bretzke ML, Lillemoe TJ. Surgical outcomes of lobular neoplasia diagnosed in core biopsy: prospective study of 316 cases. Clin Breast Cancer. 2016;16:S1526-8209.

14. Fisher B, Costantino JP, Wickerham DL, Cecchini RS, Cronin WM, Robidoux A, Bevers TB, Kavanah MT, Atkins JN, Margolese RG, Runowicz CD, James JM, Ford LG, Wolmark N. Tamoxifen for the prevention of breast cancer: current status of the National Surgical Adjuvant Breast and Bowel Project P-1 Study. JNCl. 2005:97(22):1652-62.

15. Goss PE, Ingle JN, Ales-Martinez JE, et al. Exemestane for breast-cancer prevention in postmenopausal women. N Engl J Med. 2011;364:2381-91.

16. Logan GJ, Dabbs DJ, Lucas PC, Jankowitz RC, Brown DD, Clark BZ, Oesterreich S, McAuliffe PF. Molecular drivers of lobular carcinoma in situ. Breast Cancer Res. 2015;17:76

17. Vincent-Salomon A, Hajage D, Rouquette A, Cédenot A, Gruel N, Alran S, Sastre-Garau X, Sigal-Zafrani B, Fourquet A, Kirova Y. High Ki67 expression is a risk marker of invasive relapse for classical lobular carcinoma in situ patients. Breast. 2012:21(3):380-3.

18. Giricz O, Reynolds PA, Ramnauth A, Liu C, Wang T, Stead L, Childs G, Rohan T, Shapiro N, Fineberg S, Kenny PA, Loudig O. Hsa-miR-375 is differentially expressed during breast lobular neoplasia and promotes loss of mammary acinar polarity. J Pathol. 2012;226(1):108-19.

19. Mohsin SK, O'Connell P, Allred DC, Libby AL. Biomarker profile and genetic abnormalities in lobular carcinoma in situ. Breast Cancer Res Treat. 2005 90(3):249-56.

20. Nilsen G, Liestøl K, Van Loo P, Moen Vollan HK, Eide MB, Rueda OM, Chin SF, Russell R, Baumbusch LO, Caldas C, Børresen-Dale AL, Lingjaerde OC. Copynumber: efficient algorithms for single- and multi-track copy number segmentation. BMC Genomics. 2012;13:591.

21. Reis-Filho JS, Savage K, Lambros MB, James M, Steele D, Jones RL, Dowsett M. Cyclin D1 protein overexpression and CCND1 amplification in breast carcinomas: an immunohistochemical and chromogenic in situ hybridisation analysis. Mod Pathol. 2006;19(7):999-1009.

22. Li H, Handsaker B, Wysoker A, Fennell T, Ruan J, Homer N, Marth G, Abecasis G, Durbin R. The Sequence Alignment/Map format and SAMtools. Bioinformatics. 2009;25(16):2078-9.

23. Koboldt DC, Zhang Q, Larson DE, Shen D, McLellan MD, Lin L, Miller CA, Mardis ER, Ding L, Wilson RK. VarScan 2: somatic mutation and copy number alteration discovery in cancer by exome sequencing. Genome Res. 2012;22(3):568-76.

24. Wang K, Li M, Hakonarson H. ANNOVAR: functional annotation of genetic variants from high-throughput sequencing data. Nucleic Acids Res. 2010; 38(16):164. 
25. Qiu W, Tong GX, Manolidis S, Close LG, Assaad AM, Su GH. Novel mutantenriched sequencing identified high frequency of PIK3CA mutations in pharyngeal cancer. Int J Cancer. 2008;122(5):1189-94.

26. Hui P, Howe JG, Crouch J, Nimmakayalu M, Qumsiyeh MB, Tallini G, Flynn SD, Smith BR. Real-time quantitative RT-PCR of cyclin D1 mRNA in mantle cell lymphoma: comparison with FISH and immunohistochemistry. Leuk Lymphoma. 2003;44(8):1385-94

27. Mohseni M, Cidado J, Croessmann S, Cravero K, Cimino-Mathews A, Wong HY, Scharpf R, Zabransky DJ, Abukhdeir AM, Garay JP, Wang GM, Beaver JA, Cochran RL, Blair BG, Rosen DM, Erlanger B, Argani P, Hurley PJ, Lauring J, Park BH. MACROD2 overexpression mediates estrogen independent growth and tamoxifen resistance in breast cancers. PNAS. 2014:111(49):17606-11.

28. Zhao J. KLF8: so different in ovarian and breast cancer. Oncoscience. 2014;1(4):248-9.

29. Bilal E, Alexe G, Yao M, Cong L, Kulkarni A, Ginjala V, Toppmeyer D, Ganesan $\mathrm{S}$, Bhanot $\mathrm{G}$. Identification of the YES1 kinase as a therapeutic target in basal-like breast cancers. Genes Cancer. 2010;1(10):1063-73.

30. Johnson CE, Gorringe KL, Thompson ER, Opeskin K, Boyle SE, Wang Y, Hill P, Mann GB, Campbell IG. Identification of copy number alterations associated with the progression of DCIS to invasive ductal carcinoma. Breast Cancer Res Treat. 2012;133(3):889-98.

31. Cornen S, Guille A, Adélaïde J, Addou-Klouche L, Finetti P, Saade MR, Manai M, Carbuccia N, Bekhouche I, Letessier A, Raynaud S, Charafe-Jauffret E, Jacquemier J, Spicuglia S, de The H, Viens P, Bertucci F, Birnbaum D, Chaffanet M. Candidate luminal B breast cancer genes identified by genome, gene expression and DNA methylation profiling. PLoS One. 2014; 9(1):e81843.

32. Lebok P, Mittenzwei A, Kluth M, Özden C, Taskin B, Hussein K, Möller K, Hartmann A, Lebeau A, Witzel I, Mahner S, Wölber L, Jänicke F, Geist S, Paluchowski P, Wilke C, Heilenkötter U, Simon R, Sauter G, Terracciano L, Krech R, von der Assen A, Müller V, Burandt E. 8p Deletion is strongly linked to poor prognosis in breast cancer. Cancer Biol Ther. 2015;16(7):1080-7.

33. Venkatesh T, Nagashri MN, Swamy SS, Mohiyuddin SM, Gopinath KS, Kumar A. Primary microcephaly gene MCPH1 shows signatures of tumor suppressors and is regulated by miR-27a in oral squamous cell carcinoma. PLoS One. 2013;8(3):e54643.

34. Bhattacharya N, Mukherjee N, Singh RK, Sinha S, Alam N, Roy A, Roychoudhury S, Panda CK. Frequent alterations of MCPH1 and ATM are associated with primary breast carcinoma: clinical and prognostic implications. Ann Surg Oncol. 2013;20 Suppl 3:S424-32.

35. Mantere T, Winqvist R, Kauppila S, Grip M, Jukkola-Vuorinen A, Tervasmäki A, Rapakko K, Pylkäs K. Targeted next-generation sequencing identifies a recurrent mutation in $\mathrm{MCPH} 1$ associating with hereditary breast cancer susceptibility. PLoS Genet. 2016;12(1):e1005816.

36. Castells A, Gusella JF, Ramesh V, Rustgi AK. A region of deletion on chromosome 22q13 is common to human breast and colorectal cancers. Cancer Res. 2000;60(11):2836-9.

37. Lu YJ, Osin P, Lakhani SR, Di Palma S, Gusterson BA, Shipley JM. Comparative genomic hybridization analysis of lobular carcinoma in situ and atypical lobular hyperplasia and potential roles for gains and losses of genetic material in breast neoplasia. Cancer Res. 1998;58:4721-7.

38. Courjal F, Cuny M, Simony-Lafontaine J, Louason G, Speiser P, Zeillinger R, Rodriguez C, Theillet C. Mapping of DNA amplifications at 15 chromosomal localizations in 1875 breast tumors: definition of phenotypic groups. Cancer Res. 1997;57(19):4360-7.

39. Jang M, Kim E, Choi Y, Lee H, Kim Y, Kim J, Kang E, Kim SW, Kim I, Park S. FGFR1 is amplified during the progression of in situ to invasive breast carcinoma. Breast Cancer Res. 2012;14(4):R115.

40. Burkhardt L, Grob TJ, Hermann I, Burandt E, Choschzick M, Jänicke F, Müller V, Bokemeyer C, Simon R, Sauter G, Wilczak W, Lebeau A. Gene amplification in ductal carcinoma in situ of the breast. Breast Cancer Res Treat. 2010;123(3): 757-65.

41. Shin SJ, Lal A, De Vries S, Suzuki J, Roy R, Hwang ES, Schnitt SJ, Waldman FM, Chen YY. Florid lobular carcinoma in situ: molecular profiling and comparison to classic lobular carcinoma in situ and pleomorphic lobular carcinoma in situ. Hum Pathol. 2013;44(10):1998-2009.

42. Burandt $E$, Grünert $M$, Lebeau $A$, Choschzick $M$, Quaas $A$, Jänicke $F$, Müller $V$, Scholz U, Bokemeyer C, Petersen C, Geist S, Paluchowski P, Wilke C, Heilenkötter U, Simon R, Sauter G, Wilczak W. Cyclin D1 gene amplification is highly homogeneous in breast cancer. Breast Cancer. 2016;23(1):111-9.
43. Tobin NP, Lundgren KL, Conway C, Anagnostaki L, Costello S, Landberg G. Automated image analysis of cyclin D1 protein expression in invasive lobular breast carcinoma provides independent prognostic information. Hum Pathol. 2012:43(11):2053-61.

44. Oyama T, Kashiwabara K, Yoshimoto K, Arnold A, Koerner F. Frequent overexpression of the cyclin D1 oncogene in invasive lobular carcinoma of the breast. Cancer Res. 1998;58(13):2876-80.

45. Ali HR, Rueda OM, Chin SF, Curtis C, Dunning MJ, Aparicio SA, Caldas C. Genome-driven integrated classification of breast cancer validated in over 7,500 samples. Genome Biol. 2014;15(8):431.

46. Gruel N, Lucchesi C, Raynal V, Rodrigues MJ, Pierron G, Goudefroye R, Cottu P, Reyal F, Sastre-Garau X, Fourquet A, Delattre O, Vincent-Salomon A. Lobular invasive carcinoma of the breast is a molecular entity distinct from luminal invasive ductal carcinoma. Eur J Cancer. 2010;46(13):2399-407.

47. Ciriello G, Gatza ML, Beck AH, Wilkerson MD, Rhie SK, Pastore A, Zhang H, McLellan M, Yau C, Kandoth C, Bowlby R, Shen H, Hayat S, Fieldhouse R, Lester SC, Tse GM, Factor RE, Collins LC, Allison KH, Chen YY, Jensen K, Johnson NB, Oesterreich S, Mills GB, Cherniack AD, Robertson G, Benz C, Sander C, Laird PW, Hoadley KA, King TA. TCGA Research Network, Perou CM. Comprehensive molecular portraits of invasive lobular breast cancer. Cell. 2015;163(2):506-19.

48. Sakr RA, Schizas M, Carniello JV, Ng CK, Piscuoglio S, Giri D, Andrade VP, De Brot M, Lim RS, Towers R, Weigelt B, Reis-Filho JS, King TA. Targeted capture massively parallel sequencing analysis of LCIS and invasive lobular cancer: repertoire of somatic genetic alterations and clonal relationships. Mol Oncol. 2016;10:360-70

49. Christgen M, Noskowicz M, Schipper E, Christgen H, Heil C, Krech T, Länger F, Kreipe H, Lehmann U. Oncogenic PIK3CA mutations in lobular breast cancer progression. Genes Chromosomes Cancer. 2013;52(1):69-80.

50. Li H, Zhu R, Wang L, Zhu T, Li Q, Chen Q, Wang H, Zhu H. PIK3CA mutations mostly begin to develop in ductal carcinoma of the breast. Exp Mol Pathol. 2009;88(1):150-5.

51. Ang DC, Warrick AL, Shilling A, Beadling C, Corless CL, Troxell ML. Frequent phosphatidylinositol-3-kinase mutations in proliferative breast lesions. Mod Pathol. 2014;27:740-50.

52. Andrade VP, Morrogh M, Qin LX, Olvera N, Giri D, Muhsen S, Sakr RA, Schizas M, Ng CK, Arroyo CD, Brogi E, Viale A, Morrow M, Reis-Filho JS, King TA. Gene expression profiling of lobular carcinoma in situ reveals candidate precursor genes for invasion. Mol Oncol. 2015;9:772-82.

53. Merlo LM, Shah NA, Li X, Blount PL, Vaughan TL, Reid BJ, Maley CC. A comprehensive survey of clonal diversity measures in Barrett's esophagus as biomarkers of progression to esophageal adenocarcinoma. Cancer Prev Res. 2010;3(11):1388-97.

54. Maley CC, Galipeau PC, Finley JC, Wongsurawat VJ, Li X, Sanchez CA, Paulson TG, Blount PL, Risques RA, Rabinovitch PS, Reid BJ. Genetic clonal diversity predicts progression to esophageal adenocarcinoma. Nat Genet. 2006;38(4):468-73

55. Zhang M, Rosen JM. Developmental insights into breast cancer intratumoral heterogeneity. Trends Cancer. 2015;1(4):242-51.

\section{Submit your next manuscript to BioMed Central and we will help you at every step:}

- We accept pre-submission inquiries

- Our selector tool helps you to find the most relevant journal

- We provide round the clock customer support

- Convenient online submission

- Thorough peer review

- Inclusion in PubMed and all major indexing services

- Maximum visibility for your research

Submit your manuscript at www.biomedcentral.com/submit 